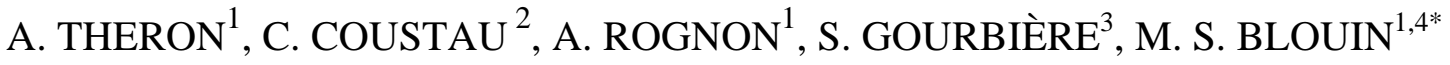
9

${ }^{1}$ UMR 5244 CNRS-EPHE-UPVD, Biologie et Ecologie Tropicale et Méditerranéenne,

Université Via Domitia. 52 Av. Paul Alduy. 66860 Perpignan Cedex, France.

13

${ }^{2}$ U547 Inserm, Institut Pasteur de Lille, 1 Rue du Prof. Calmette, BP 245. 59019 Lille Cedex,

France.

16

${ }^{3}$ EA 3680, Mathématiques Et Physique pour les Systèmes (MEPS), Université de Perpignan

Via Domitia, 52 Av. Paul Alduy. 66860 Perpignan Cedex, France.

19

${ }^{4}$ Dept. Zoology, Oregon State University, Corvallis, OR, 97331 USA

21

22

*Corresponding author: Tel: 541-737-2362; Fax : 541-737-0501; email:

24 blouinm@science.oregonstate.edu

25

26

Running title: schistosome-snail compatibility

27 
29 The genetic control of compatibility between laboratory strains of schistosomes and their snail hosts has been studied intensively since the 1970s. These studies show (1) a bewildering array of genotype-by-genotype interactions - compatibility between one pair of strains rarely predicts compatibility with other strains, and (2) evidence for a variety of (sometimes conflicting) genetic mechanisms. Why do we observe such variable and conflicting results? One possibility is that it is partly an artifact of the use of laboratory strains that have been in culture for many years and are often inbred. Here we show that results of compatibility trials between snails and schistosomes - all derived from the same natural population - depend very much on whether one uses laboratory-cultured or field-collected individuals. Explanations include environmental effects of the lab on either host or parasite, and genetic changes in either host or parasite during laboratory culture. One intriguing possibility is that genetic bottlenecks during laboratory culture cause the random fixation of alleles at highly polymorphic loci that control the matched/mismatched status of hosts and parasites. We show that a simple model of phenotype matching could produce dose response curves that look very similar to empirical observations. Such a model would explain much of the genotype-bygenotype interaction in compatibility observed among strains.

Keywords: Schistosoma mansoni, Biomphalaria glabrata, resistance, susceptibility, 48 compatibility, parasite, host 
Compatibility in host-parasite systems can be defined as the state in which a parasite can establish infection and complete its development in a host (Basch, 1975). Thus, compatibility is a joint trait of the parasite and host that probably depends on the genotypes of parasite and host at many different loci. Consequently, there is ample opportunity for complex genotypeby-genotype interactions: the phenotype of the host (susceptible/resistant) depends on the genotype of the parasite, and the phenotype of the parasite (infective/noninfective) depends on the genotype of the host (Lambrechts et al., 2006). Genotype-by-genotype interaction is particularly apparent in the literature on compatibility between schistosomes and their snail first intermediate hosts (Richards and Shade, 1987; Morand et al., 1996; Webster et al.,2004; Theron and Cousteau, 2005). As an agent of human schistosomiasis, Schistosoma mansoni and its new world snail host, Biomphalaria glabrata, have been the subjects of numerous studies on the genetic basis of variation in compatibility between different laboratory strains of snails and parasite (here we use the term "strain" to refer to a field isolate maintained in the laboratory for at least one generation). One consistent result, in study after study, is that snail strains that are naturally or selected to be highly incompatible with one particular schistosome strain, often remain highly compatible with other schistosome strains (Richards and Shade, 1987; Webster and Woolhouse, 1998; Webster et al., 2004). Susceptibility to particular schistosome strains can be highly heritable in snails (Richards and Shade, 1987; Richards et al., 1992; Webster et al., 2004). However, attempts to analyze the genetic basis of differences among particular snail strains in their susceptibility to a given schistosome strain have suggested a variety of mechanisms, including single or multi-locus inheritance, and either dominance or recessiveness of the trait (Richards, 1975; Richards et al., 1992; Webster 2001). To date, only one candidate locus has been found to associate with compatibility in either parasite or host (Goodall et al., 2006; Bender et al., 2007).

Here we present compatibility studies conducted using lab and field samples of snails and schistosomes, all derived from the same natural population in Guadeloupe, West Indies. We show that results of compatibility trials depend very much on whether one uses laboratorycultured or field-collected individuals. We lay out possible hypotheses to explain some of these results in the hopes of stimulating new research directions. 
Brief overview of experiments

We tested snail-schistosome compatibility through four main types of experiments (table 1) : (i) compatibility trails using lab strains of snails and schistosomes ; (ii) compatibility trails using wild snails and wild schistosomes; (iii) compatibility trials involving first wild snails and parasites and later their corresponding snails and schistosomes after each of the first three generations of lab culture; finally (iv) compatibility trials using lab snails challenged by wild schistosomes.

\section{Sampling site}

All host and parasite samples in this study originated from the same transmission site at Dans Fond (DFO; N:16 $\left.18.500^{\prime}, \mathrm{W}: 061^{\circ} 30.720^{\prime}\right)$, located in the marshy forest of Grande Terre Island in Guadeloupe, West Indies (Theron and Pointier, 1995). This site has been the subject of numerous studies on the ecology, dynamics and genetics of larval and adult S. mansoni, their intermediate host, B. glabrata, and their definitive host, Rattus rattus (Sire et al., 1999; 2001 a,b; Theron et al., 2004; Prugnolle et al., 2005a,b; 2006). Transmission is characterized here by very low prevalence in snails ( $0.6 \%$ on average), contrasting with very high infection rates (94\%) and heavy schistosome loads (160 worms per rat on average) within the definitive hosts.

\section{Measuring compatibility: snail exposure, infection rates and intensities}

The level of compatibility for a particular snail-schistosome combination is traditionnally quantified as the proportion of snails infected after individual exposure to a fixed number of miracidia, (usually in the range of 5-20 miracidia). Snail infection rates vary with the parasite dose used (Theron et al., 1997), which makes it difficult to compare the outcomes of different studies. Therefore, we evaluated compatibility between host and parasite populations or strains by challenging individual snails with different numbers of miracidia. Although such dose-response curves are labor-intensive to produce, they are much more informative about the dynamics of compatibility between two strains than are single-dose challenges. All challenge experiments described in this study were conducted by the same person (A. Rognon), using the same protocols. For each experiment, snails (10-12 mm in diameter) were exposed individually to a fixed number of miracidia in approximately $10 \mathrm{ml}$ of water for 8 hours. Following exposure to miracidia, snails were replaced in their original containers until their infection status was assessed. 
117 The infected or uninfected status of the exposed snails was detected by two different methods : (i) the shedding of cercariae 30 days post-exposure (the length of the prepatent period); (ii) the presence of well developed mother sporocysts (MSp) in the head foot region 15 days post exposure. For the detection of mother sporocysts, the snails were fixed 15 days post-exposure following the methods described by Theron and Gerard (1994). In brief, snails were relaxed in pond water containing an excess crystalline menthol for 12 hours. The snail body was removed and fixed in modified Raillet-Henry's solution. The number of MSp present in each snail was determined following exhautive dissection of the head-foot zone. In this technique, MSp's were readily observable as translucent white bodies within an opaque grey tissue background (Fig. 1). For field-collected snails, the technique also allowed us to distinguish a posteriori between snails infected during the experiment from those naturally infected but undetectable because they were in the prepatent period during sampling. This distinction is easily made by the presence of young daughter sporocysts in the hepatopancreas of the snails at the time of dissection.

\section{Compatibility trials using laboratory strains}

Laboratory strains of snails and of schistosomes have been isolated from Dans Fond and established in our laboratory at the University of Perpignan three times: in 1992, 2000 and 2005 (Table 1).

The snail strains were founded each time using 100-150 uninfected founders from the field. The snail populations were allowed to expand quickly and were then maintained at a census size in the hundreds of individuals. Snails in the lab are allowed to breed freely, and the lab populations are not exposed to any deliberate selection. Note that although B. glabrata are hermaphroditic, they preferentially outcross and show Hardy-Weinberg equilibrium at molecular markers in both laboratory and field populations (Prugnolle et al., 2005b; unpub. data).

The schistosomes strains were founded each time using cercariae from 43-47 infected snails previously exposed to 20 miracidia hatched from eggs collected from the livers of six naturally-infected $R$. Rattus. In the lab, schistosome strains are passaged each generation through five mice and approximately 24 infected snails (8-10 miracidia at exposure). 
150 Compatibility trails were performed for snail and parasite strains established in 1992 and 2005 and tested after 5 years (Theron et al., 1997) and 2 years maintenance in the lab, respectively (labelled as “LAB 1997” and “LAB 2007” in Fig. 2A). Dose-response curves were obtained by challenging individual snails (45-50 snails per treatment) with doses of 1,2 , 5, 10, 20, 30 and 50 miracidia. The doses of 20 and 5 miracidia were omitted for the LAB 1997 and LAB 2007 experiments, respectively. For both experiments snail infections rates and parasite intensities were evaluated by mother sporocyst count.

\section{Compatibility trials using wild schistosomes and wild snails}

In 2000 and 2005 we generated dose-response curves using schistosomes and snails collected directly from the transmission site (i.e. never passaged in the lab). We refer to these experiments as “DFO 2000” and “DFO 2005”. All challenges were conducted the INRADuclos laboratory in Guadeloupe. The livers of heavily infected rats were crushed in a saline solution, and the homogenates were passed through different filters to finally retrieve only schistosome eggs. For each experiment, schistosome eggs were collected from six heavily infected rats ( $>100$ worms/host) and placed in fresh water to hatch into miracidia.

Dose-response curves were obtained by challenging individual snails (50 snails per treatment) with doses of 1, 2, 10, 20, 30 and 50 miracidia (Fig. 2B). For the DFO 2000 experiment, all the snails were fixed 15 days post exposure and dissected to count numbers of mother sporocysts per snail. Dissections showed no evidence of pre-patent infections in any of our field-collected snails. Also, the prevalence of infection in the field averages only $0.6 \%$.

Therefore we used the less-laborious cercarial shedding test for the DFO 2005 experiment on the assumption that pre-patent infections would be so rare as to not appreciably influence our results.

\section{Compatibility during the first three generations of laboratory passage}

In this experiment we followed the change in compatibility between snails and parasites in going from the field through the first three generations of passage in the laboratory. Here we used a single challenge dose (20 miracidia), rather than generating a dose-response curve each time. In 2000, one hundred field-collected snails were individually exposed to 20 wild miracidia. Fifty of these snails were transported back to our laboratory in France, and cercariae shed by 43 of the 46 survivors were used to infect mice and establish a schistosome strain. The remaining 50 wild exposed snails were fixed and dissected to measure infection 
184

185

186

187

188

189

190

191

192

193

194

195

196

197

198

199

200

201

202

203

204

205

206

207

208

209

210

211

212

213

214

215

rates and numbers of mother sporocysts per snail. The lab strain of snails was established from 100 uninfected, field-collected founders. After each of the first three generations of culture in France, 50 lab snails were each challenged with 20 lab miracidia. These were also fixed and dissected to measure infection rates and numbers of mother sporocysts per snail.

The lab strains isolated in 2005 were tested in the field, and then after each generation of parasite passage, using a dose of 20 miracidia. We have continued testing them each generation, using the same dose.

\section{Compatibility trials using wild versus laboratory snails or parasites}

It would be ideal to also have replicated trials in which lab parasites were used to challenge field snails, and field parasites used to challenge lab snails. Owing to logistical constraints the only combination we were able to attempt was a single trial in which lab snails were challenged with field parasites. After the laboratory strain of snail established in 2000 had been in captivity for two years, 300 of these lab snails were transported to Guadeloupe to be challenged with wild miracidia. We generated a dose-response curve using these snails and the same protocols as for the wild-by-wild combinations. Infection rates and intensities (number of mother sporocysts per snail) were measured by dissecting each snail. Because we have not been able to replicate this experiment, we consider the results of this trial to be preliminary. We present them here simply for the sake of completeness.

\section{RESULTS}

\section{Laboratory schistosomes versus laboratory snails}

The dose-response curve generated using the 1992 laboratory strains shows a rapid increase in infection rate up to a dose of 10-20 miracidia, but then levels off at about $65 \%$ infection (LAB 1997 in Fig. 2A). This result suggests that about 35\% of the snails in the laboratory population were completely resistant to infection, regardless of the number of miracidia used to challenge. A very similar result was obtained with the 2005 lab isolates, except the curve leveled off at about 45\% infection (LAB 2007 in Fig. 2A).

\section{Wild schistosomes versus wild snails}


216 Results obtained for the two wild-by-wild dose-response curves (experiments DFO 2000 and

217 DFO 2005) are shown in Fig. 2B. Infection rates increased rapidly with increasing dose,

218 reaching $90-100 \%$ at $20-30$ miracidia, and $100 \%$ at the highest dose.

Change in compatibility in going from field isolates to laboratory strains

Among the 50 snails that were each exposed to 20 miracidia in Guadeloupe in 2000, 46 survived and 43 of those became infected (93.5\%), shed cercariae, and were used to found the schistosome colony in our lab in France. Among the 50 other snails exposed in the same conditions and fixed, 48 harbored developed MSp's (96\%). The compatibility of this pair of strains using a dose of 20 miracidia dropped to 51.0\%, 59.8\% and $54.1 \%$ after 1, 2, and 3 passages through laboratory mice, respectively (Fig. 3). A similar pattern occurred with the strains isolated in 2005. Again, infection rates dropped from near 100\% when wild snails were challenged with 20 wild miracidia apiece, to around $40 \%$ after a single generation of passage in the lab. We continue to check the infection rate after each passage, and it remains at about $40 \%$ to this day (mean \pm 1 s.e. $=41.2 \pm 2.8$ ) - right in line with the results of the dose-response curve (Fig. 2A).

Wild schistosomes versus laboratory snails

Infection rates of laboratory bred snails exposed to wild miracidia increased gradually to 95\% at the highest dose (Fig. 2C). Interestingly, the shape of this curve differs from those of the wild-by-wild or lab-by-lab curves. Rather than quickly reaching a plateau, the infection rate increases almost linearly. So this result seems intermediate between those of the lab-by-lab and field-by-field trials. In this case lab snails appeared to be much more susceptible to groups of wild miracidia than to the same number of lab miracidia, yet not as susceptible as wild snails.

\section{Infection intensities (establishment of mother sporocysts)}

243 In the lab-by-lab trials, infection intensities rose gradually and then leveled off at 244 approximately 2-3 /snail after challenge by 10 or more miracidia (Fig. 2A). In contrast, in the wild-by-wild challenge the mean number of mother sporocysts (MSp) that developed within infected snails increased steadily to an average of $6.6 \pm 1.3 \mathrm{MSp} / \mathrm{snail}$ at the 50 miracidial dose (Fig. 2B), with a maximum of $16 \mathrm{MSp}$ found in one snail. Infection intensities in the wild-schistosome by lab-snail trials (Fig. 2C) were lower than in the field-by-field trials, reaching a maximum of 3.4 MSp/snail at a miracidial dose of 50. 
Figure 3 shows the change in number of MSp's per infected snail during the first three generations of passage of the 2000 lab strains. Although a dose of 20 wild miracidia produced an average of 5.37 MSp per infected snail in Guadeloupe, that number dropped to 2.04, 1.92 and 1.63 MSp/snail over three generations of passage in the lab. Thus, as with the percentage of snails infected, the number of sporocysts per infected snail dropped precipitously after the first generation of passage, and then remained relatively constant thereafter.

\section{DISCUSSION}

\section{Loss of compatibility in going from field to lab}

In the year-2000 field trials a challenge with 20 wild miracidia produced 95\% infection. Yet after a single generation of passage in the lab (of schistosome through the new lab snails) the percent infection dropped to $51 \%$ and remained between $54 \%$ and $60 \%$ for the next two generations. The same result was obtained when we established new strains of parasite and snail in 2005 - almost $100 \%$ infection in the field dropped to about $40 \%$ after a single passage in lab, and stayed the same thereafter. The number of MSp established per infected snail also drops after laboratory passage (Fig. 3).

Thus it appears that in three independent isolations of schistosomes and snails from the same site, there was a massive drop in compatibility in going from field to lab. Even more intriguing is the observation that in two independent isolations of snails and parasites from the field, it appears that a large fraction of the lab snails became completely resistant to the lab strain of schistosomes (Fig. 2A). In stark contrast, we see $100 \%$ compatibility when wild snails are challenged with enough wild miracidia (above 20- 30 miracidia), and much higher rates and intensities of infection at the lower doses (Fig. 2B).

There are three main hypotheses to explain these results. (1) Experimental artifact: the lab-vslab trials were conducted in France, and the field-by-field trials were conducted in Guadeloupe. Thus some experimental factor might have been consistently different between the two settings. (2) Environmental effects: something about the laboratory environment makes the parasite less infective and/or the snails more resistant. (2) Genetic effects: either the snail population, the parasite population, or both underwent genetic changes during laboratory culture. 


\section{Experimental artifact}

We took great pains to insure that the trials conducted in the lab in Guadeloupe were as similar as possible to the trials conducted in the lab in France (same person did all the trials, same equipment, and so on). Regardless, it is difficult to imagine how experimental artifact could produce the plateaus seen in the lab-by-lab curves, which suggest the existence of a completely resistant subset of the lab snail population. For example, if some factor (say the water) reduced the average infectivity of miracidia in the lab in France (or resistance of the snails), then the lab-by-lab curves would have shapes similar to those of the field-by-field curves, but simply with a lower inflection point. Thus, we think that the fundamentally different shapes of the two sets of curves really does reveal an interesting biological phenomenon that deserves further study.

\section{Environmental effects}

It is well known that the susceptibility of snails can depend on, for example, their size, age and physiological status (Anderson et al., 1982; Theron et al., 1998; Krist et al., 2004). So it is quite plausible that something about being raised in the lab environment makes snails more resistant. For example, perhaps nutritional status differs or some microbe or other stressor in the lab tank keeps the immune system of lab snails ramped up (Hertel et al. 2002). But again, it is difficult to imagine a mechanism that would generate a completely resistant subset of the population, rather than just a higher average resistance per snail. An environmental effect on the parasite is also possible, although again we do not have any candidate mechanism that would render that strain of parasites completely unable to infect just a subset of the snail population.

\section{Genetic effects}

(1) Genetic change in the snails: The lab breeding colony of snails is never challenged, so any selected change must have been a correlated response to selection on some other trait that is inadvertently under selection in the lab. Genetic drift remains a possibility, but the lab colony was founded each time using more than 100 individuals, and remained large thereafter. Also, lab strains of B. glabrata tend to retain substantial molecular genetic diversity (e.g. Mulvey and Vrijenhoek, 1981; Campos et al., 2002). Thus, it is hard to believe that just one or two generations of drift in the snails caused the changes we observed (e.g. Fig. 3). 
(2) Genetic change in the schistosomes: If selection is involved, one possible source is the

318 definitive host. Rats are the definitive host in nature, but the parasites are passed through mice in the lab. It would be quite interesting if selection for performance in a novel definitive host resulted in a correlated response in infectivity to snails. Such a result would suggest that the parasite uses some common mechanism to infect both the intermediate and definitive hosts.

An even more plausible genetic explanation is rapid genetic drift in the lab schistosome isolate. The miracidium that infects a snail becomes a sporocyst that then undergoes a round of asexual reproduction that culminates in the release of thousands of genetically identical cercariae. In most labs, rodents are infected by being placed in water that contains cercariae shed by several infected snails. Thus, there is a huge potential for non-random variance among clones in transmission to the next generation if care is not taken to equalize the cercarial contribution from each snail. Strong selection among clones in going from the natural definitive host (rats or humans) to mice or hamsters in the lab would add an additional, non-random component to the among-clone variance in transmission success (e.g. we see high variance among clones in their inherent infectivity to mice; J. Boissier, pers. comm.). Rodents are expensive to maintain, so most lab strains of S. mansoni are passed through only a handful of rodents each generation. A mouse can support 50-100 adult schistosomes at most. We have little idea what is the variance in family size (egg production) among adult worms, and thus what is the effective number of breeders per host that contributes to the next generation (Criscione and Blouin, 2005). Thus, most lab strains of schistosomes probably undergo severe genetic bottlenecks beginning with the first generation of lab passage. Consistent with this prediction, molecular genetic studies show that lab strains of schistosomes have only a fraction of the allelic variation found in field samples, and they also show the distorted allele frequency distributions typical of bottlenecked populations

343 (Cornuet and Luikart, 1997; Stohler et al., 2004).

\section{A possible mechanism: matching alleles and bottlenecks}

346 One hypothesis that would explain our results is that the success or failure of a challenge by one parasite depends on the matched/mismatched status of the host and parasite genotypes, as in matching-alleles models (Agrawal and Lively, 2002; Basch, 1975; Theron and Coustau, 2005). Any one snail and miracidium may have a modest probability of matching. However, as a snail is challenged with increasing numbers of miracidia, the probability of at least one 
match increases rapidly. Increasing the miracidial dose simply involves sampling a larger fraction of the phenotypic diversity in the parasite population. Under this model all snails eventually succumb when challenged by enough wild miracidia because the probability of at least one match approached 1.0. Such a system of phenotype matching could then explain the drop in compatibility after laboratory passage if the phenotypic variation is genetically based. If laboratory passage involves a more severe genetic bottleneck and loss of alleles in the parasite population than in the snail population, then a large fraction of the snails could quickly become "resistant", owing simply to loss of compatible alleles in the schistosome strain. Under this scenario those snails are not "resistant” to schistosomes per se. They are simply not matched by any individuals in the now genetically-depauperate lab strain of parasites.

Such a genetically-based system of phenotype matching could generate curves that look very much like those in figure $2 \mathrm{~A}$ and $2 \mathrm{~B}$. If each miracidium has a low, but constant probability of matching a target snail, and if miracidia infect independently of each other, then the probability that a snail becomes infected when challenged by $x$ miracidia can be written

(1) $\mathrm{P}($ infection $)=\left(1-(1-p)^{x}\right)$,

where $p=$ the probability of infection per miracidium. For example, in figure 4A we plotted equation 1 with a value of $p=0.11$ in the same panel as the wild-vs.-wild data from 2002. Curves like those in Fig. 2B, in which there appears to be a completely resistant subset of snails, can be generated simply by multiplying equation 1 by a constant, $f$, which is the fraction of snails that can be infected. For example, in figure 4B we plotted the 1997 lab-vs.lab data and equation (1) with $f=0.67$ and $p=0.2$.

One can also generate similar-looking curves via a matching genotypes model. Here the snail becomes infected if at least one miracidium in the pool of challengers carries the same (matching) genotype as the snail. The probability that a snail becomes infected when challenged by $x$ miracidia equals

(2) $\mathrm{P}($ infection $)=f \sum_{i=1}^{N_{a}}\left(h_{i}\right)\left(1-\left(1-p_{i}\right)^{x}\right)$, 
where $N_{a}=$ the number of genotypes present in the parasite population, $h_{i}=$ the frequency of genotype $i$ in the host population, $p_{i}=$ the frequency of genotype $i$ in the parasite population. If all snail alleles have a matching allele in the parasite population, then these curves will asymptote to $100 \%$ infection as $x$, the number of challenging miracidia, increases. If some snail genotypes are not matched by a genotype in the parasite population, then the curve will asymptote at $f$, the fraction of snails that can be matched.

The actual shapes of curves generated by equation 2 will obviously depend on the particular allele frequency distributions in parasite and host populations. We can, a posteriori, choose parameter values that make the curves from equation 3 fit some of our observed data quite well (Fig. 4). This does not mean we have proof for a matching genotypes model, but it does show that such a model remains a plausible explanation for our results.

Some possible consequences of using bottlenecked lab strains

If the bottleneck hypothesis is correct, then this could explain why studies to date show such a variety of genetic mechanisms and of strain-by-strain interactions. The chance loss or fixation of alleles at compatibility loci may cause different loci to appear overly important in controlling compatibility in different pair-wise combinations. In other words, in different studies a different set of loci may, by chance, explain a disproportionate amount of the variance in compatibility between particular lab strains. Loci that might be particularly misleading in that sense include loci encoding highly polymorphic systems of matching alleles that hosts use to recognize invaders, and/or invaders use to mimic hosts (Zhang et al., 2004; Theron and Coustau 2005). Here the compatibility of any pair-wise combination could depend largely on which matching alleles were lost by chance during the domestication of each partner, and so would not predict compatibility with other strains, or with the original field populations. For example, this hypothesis predicts that if we had selected the apparently resistant fraction of snails revealed in Figure 2A, we would have rapidly obtained a snail strain that is highly "resistant" to that particular schistosome strain (we are currently conducting that experiment using the 2005 strains). However, in this case the proportion of snails that did not acquire infection would not be sensus stricto resistant to S. mansoni. They

414 would simply be not matched by the reduced number of parasite genotypes contained within that particular bottlenecked laboratory strain of schistosome. Those snails might remain 
highly compatible with other schistosome strains that retain different alleles (cf Theron \&

417 Coustau, 2005).

418

419 We are not arguing that all variation in compatibility results from polymorphic systems of 420 matching alleles. Compatibility is a complex process that involves many steps from

421 recognizing the invader to preventing its successful establishment (Loker et al., 2004).

422 Indeed, the only resistance locus identified to date (Goodall et al., 2006; Bender et al., 2007)

423 is clearly part of an effector mechanism - killing the parasite once it has been successfully

424 recognized by the host. But the idea that using inbred strains causes different loci to be 425 important in each strain-by-strain comparison would hold for any polymorphic, genetically426 based mechanism of resistance.

\section{Some caveats}

429 We recognize that replicated trials of each reciprocal combination of field-by-lab would have 430 gone a long way towards identifying which species is responsible for the difference between 431 lab and field trials. The one combination we were able to attempt (lab-snails versus field432 schistosomes) produced intriguing results in that infection rates were, overall, lower than in 433 the wild-by-wild combination, but there was no plateau. The higher infection rates are 434 consistent with the bottleneck hypothesis. But if parasite diversity was all that mattered, the 435 curve should have looked just like the field-by-field curves, not the more gradual increase 436 with dose that we observed. Taken at face value, this result suggests that something about the 437 snails also changed in the lab. But until this result can be replicated, we hesitate to make too 438 much of it.

Summary

441 To our knowledge, this is the first study to compare the results of compatibility trials using

442 lab-reared and field-collected individuals from the same source populations. We showed that 443 even a single generation of laboratory passage had a large effect on the results of 444 compatibility trials. Barring experimental artifact, possible explanations include 445 environmental effects on either host or parasite, and genetic change in either host or parasite.

446 We suggest one possible mechanism that could produce curves very much like those 447 observed. The bottleneck hypothesis could explain much of the highly variable strain-by448 strain compatibilities that are so apparent in the literature on schistosomes and their snail 449 hosts. 
451 ACKNOWLEDGEMENTS

452 We thank Hervé Mauleon (INRA, Domaine Duclos) for logistical support in Guadeloupe, and 453 Jean-Pierre Pointier for help with field samples. This work received financial support from 454 the CNRS (EDD), and the Ministère de l'Ecologie et du Développment Durable (Ecosystèms 455 Tropicaux $n^{0}$ 752600). Thanks to C. Bayne, J. Tennessen, C. Adema and M. Steinauer for 456 comments on earlier drafts. M. Blouin's sabbatical at CNRS-UPVD was supported by the 457 University of Perpignan and Oregon State University. 
Agrawal, A. F. and Lively, C. M. (2003). Modelling infection as a two-step process combining gene-for-gene and matching-allele genetics. Proceedings. Biological sciences / The Royal Society 270, 323-334. Schistosoma mansoni from man to snail: experimental studies of miracidial survival and infectivity in relation to larval age, water temperature, host size and host age. Parasitology 85 (Pt 2), 339-360.

Basch, P. F. (1975). An interpretation of snail-trematode infection rates: specificitity based on concordance of compatible phenotypes. Inernationalt Journal for Parasitology 5, 449-452. Bayne, C. J., Hahn, U. K. and Bender, R. C. (2001). Mechanisms of molluscan host resistance and of parasite strategies for survival. Parasitology 123 Suppl, S159-167. variability of the main intermediate host of the Schistosoma mansoni in Brazil, Biomphalaria glabrata (Gastropoda: Planorbidae) assessed by SSR-PCR. Acta Tropica 83, 19-27. Cornuet, J. M. and Luikart, G. (1996). Description and power analysis of two tests for detecting recent population bottlenecks from allele frequency data. Genetics 144, 2001-2014. Criscione, C. D. and Blouin, M. S. (2005). Effective sizes of macroparasite populations: a conceptual model. Trends in Parasitol 21, 212-217.

478 Goodall, C. P., Bender, R. C., Brooks, J. K. and Bayne, C. J. (2006). Biomphalaria glabrata cytosolic copper/zinc superoxide dismutase (SOD1) gene: association of SOD1 alleles with resistance/susceptibility to Schistosoma mansoni. Molecular and Biochemical Parasitology 147, 207-210.

Hertel, L. A., Bayne, C. J. and Loker, E. S. (2002). The symbiont Capsaspora owczarzaki, nov. gen. nov. sp., isolated from three strains of the pulmonate snail Biomphalaria glabrata is related to members of the Mesomycetozoea. International Journal for Parasitology 32, 11831191.

Krist, A. C., Jokela, J., Wiehn, J. and Lively, C. M. (2004). Effects of host condition on susceptibility to infection, parasite developmental rate, and parasite transmission in a snailtrematode interaction. Journal of Evolutionary Biology 17, 33-40.

489 Lambrechts, L., Fellous, S. and Koella, J. C. (2006). Coevolutionary interactions between 490 host and parasite genotypes. Trends in Parasitol 22, 12-16. 
491

492

493

494

495

496

497

498

499

500

501

502

503

504

505

506

507

508

509

510

511

512

513

514

515

516

517

518

519

522

520 Sire, C., Durand, P., Pointier, J. P. and Theron, A. (2001a). Genetic diversity of

521 Schistosoma mansoni within and among individual hosts (Rattus rattus): infrapopulation

Loker, E. S., Adema, C. M., Zhang, S. M. and Kepler, T. B. (2004). Invertebrate immune systems--not homogeneous, not simple, not well understood. Immunological Reviews 198, 1024.

Morand, S., Manning, S. D. and Woolhouse, M. E. (1996). Parasite-host coevolution and geographic patterns of parasite infectivity and host susceptibility. Proceedings. Biological sciences / The Royal Society 263, 119-128.

Mulvey, M. and Vrijenhoek, R. C. (1981). Genetic variation among laboratory strains of the planorbid snail Biomphalaria glabrata. Biochemical Genetics 19, 1169-1182.

Prugnolle, F., De Meeus, T., Pointier, J. P., Durand, P., Rognon, A. and Theron, A. (2006). Geographical variations in infectivity and susceptibility in the host-parasite system Schistosoma mansoni/Biomphalaria glabrata: no evidence for local adaptation. Parasitology 133, 313-319.

Prugnolle, F., Liu, H., De Meeus, T. and Balloux, F. (2005a). Population genetics of complex life-cycle parasites: an illustration with trematodes. International Journal For Parasitology 35, 255-263.

Prugnolle, F., Theron, A., Pointier, J. P., Jabbour-Zahab, R., Jarne, P., Durand, P. and De Meeus, T. (2005b). Dispersal in a parasitic worm and its two hosts: consequence for local adaptation. Evolution Int J Org Evolution 59, 296-303.

Richards, C. S. (1975). Genetic factors in susceptibility of Biomphalaria glabrata for different strains of Schistosoma mansoni. Parasitology 70, 231-241.

Richards, C. S., Knight, M. and Lewis, F. A. (1992). Genetics of Biomphalaria glabrata and its effect on the outcome of Schistosoma mansoni infection. Parasitology Today 8, 171174.

Richards, C. S. and Shade, P. C. (1987). The genetic variation of compatibility in Biomphalaria glabrata and Schistosoma mansoni. Journal of Parasitol 73, 1146-1151.

Sire, C., Durand, P., Pointier, J. P. and Theron, A. (1999). Genetic diversity and recruitment pattern of Schistosoma mansoni in a Biomphalaria glabrata snail population: a field study using random-amplified polymorphic DNA markers. Journal of Parasitol 85, 436441. differentiation at microspatial scale. International Journal for Parasitology 31, 1609-1616. 
Sire, C., Langand, J., Barral, V. and Theron, A. (2001b). Parasite (Schistosoma mansoni) and host (Biomphalaria glabrata) genetic diversity: population structure in a fragmented landscape. Parasitology 122, 545-554. polymorphism and heterozygosity among field and laboratory populations of Schistosoma mansoni. International Journal for Parasitology 34, 595-601.

Theron, A. and Coustau, C. (2005). Are Biomphalaria snails resistant to Schistosoma mansoni? Journal of Helminthology 79, 187-191.

Theron, A. and Gerard, C. (1994). Development of accessory sexual organs n Biomphalaria glabrata as related to infection timing by Schistosoma mansoni: Consequences on the energy utilisation patterns by the parasite. The Journal of Molluscan Studies 60, 78-85.

Theron, A., Pages, J. R. and Rognon, A. (1997). Schistosoma mansoni: distribution patterns of miracidia among Biomphalaria glabrata snail as related to host susceptibility and sporocyst regulatory processes. Experimental Parasitology 85, 1-9. Theron, A. and Pointier, J. P. (1995). Ecology, dynamics, genetics and divergence of trematode populations in heterogeneous environments: the model of Schistosoma mansoni in the insular focus of Guadeloupe. Research Reviews in Parasitology 55, 49-64.

Theron, A., Rognon, A. and Pages, J. R. (1998). Host choice by larval parasites: a study of Biomphalaria glabrata snails and Schistosoma mansoni miracidia related to host size. Parasitology Research 84, 727-732.

543 Theron, A., Sire, C., Rognon, A., Prugnolle, F. and Durand, P. (2004). Molecular ecology 544 of Schistosoma mansoni transmission inferred from the genetic composition of larval and 545 adult infrapopulations within intermediate and definitive hosts. Parasitology 129, 571-585.

546 Webster, J. P. (2001). Compatibility and sex in a snail-schistosome system. Parasitology 547 122, 423-432.

548 Webster, J. P., Gower, C. M. and Blair, L. (2004). Do hosts and parasites coevolve? 549 Empirical support from the Schistosoma system. American Naturalist 164 Suppl 5, S33-51.

550 Webster, J. P., Woolhouse, M.E.J. (1998). Selection and strain specificity of compatibility 551 between snail intermediate hosts and their parasitic schistosomes. Evolution Int J Org 552 Evolution 52, 1627-1634.

553 Zhang, S. M., Adema, C. M., Kepler, T. B. and Loker, E. S. (2004). Diversification of Ig 554 superfamily genes in an invertebrate. Science 305, 251-254. 


\begin{tabular}{|l|l|l|l|l|}
\hline $\begin{array}{l}\text { Strains } \\
\text { compared }\end{array}$ & $\begin{array}{l}\text { Year strains } \\
\text { isolated from } \\
\text { field }\end{array}$ & Year tested & $\begin{array}{l}\text { Dose-response } \\
\text { curve done? }\end{array}$ & $\begin{array}{l}\text { Method used to } \\
\text { verify infection }\end{array}$ \\
\hline lab-vs-lab & 1992 & 1997 & Yes & Dissection \\
\hline & 2000 & 2000 & No $^{1}$ & Dissection \\
\hline & 2005 & 2007 & Yes $^{2}$ & Dissection \\
\hline wild-vs-wild & NA & 2000 & Yes & Dissection \\
\hline $\begin{array}{l}\text { wild parasites- } \\
\text { vs-lab snails }\end{array}$ & 2000 (snails) & 2002 & Yes & Yhedding \\
\hline
\end{tabular}

558

559

560

561

562

563

564

${ }^{1}$ challenges using 20 miracidia performed for first three generations of laboratory passage.

${ }^{2}$ challenges using 20 miracida performed every generation since the strains were established ${ }^{3}$ snails were either fixed and dissected to count number of mother sporocysts (Dissection) or were observed for cercarial shedding 30 days post exposure (Shedding). 


\section{Figure legends}

566 Fig. 1. Head-foot region of a Biomphalaria glabrata fixed 15 days post-exposure to 20 miracidia of Schistosoma mansoni. Six well developed mother sporocysts of Schistosoma mansoni are easily observable as white bodies within the snail tissue.

569

Fig. 2. Infection rates ( \pm one standard error) of individual snails and mean number (bar \pm one standard error) of mother sporocysts (MSp) per infected snail exposed to increasing doses of

572 Schistosoma mansoni miracidia (nMi). (A) Both parasites and snails were maintained in lab 573 for 5 years. $\mathrm{N}=45$ snails per dose. (B) Wild snails challenged with wild miracidia. Data 574 from 2 different trials in the DFO site. $\mathrm{N}=39-50$ surviving snails per dose. MSp data were 575 collected only in 2000. (C) Laboratory snails maintained for two years and then challenged 576 by wild miracidia. $\mathrm{N}=39-45$ surviving snails per dose.

578 Fig. 3. Snail infection rates (grey bar \pm one standard error) and mean number (black bar \pm 579 one standard error) of mother sporocysts (MSp) per infected snail after exposure to 20

580 miracidia. Snails and parasites were both obtained from the wild population (Field) in 2000, 581 and were then tested after 1, 2, and 3 laboratory generations of passage.

582

583 Fig. 4. Example of how simple models of independent infection with low individual infection 584 probabilities can generate curves that look very similar to some empirical curves. (A) 2000 585 wild-by-wild data (circles) versus equation 1 parameterized with $\mathrm{p}=0.11$ (line). (B) 1997 586 lab-by-lab data (circles) versus equation 2 parameterized by $f=0.67$ and $p=0.2$ (line). 
587

588

589

590

591

592

593

594

595

596

597

598

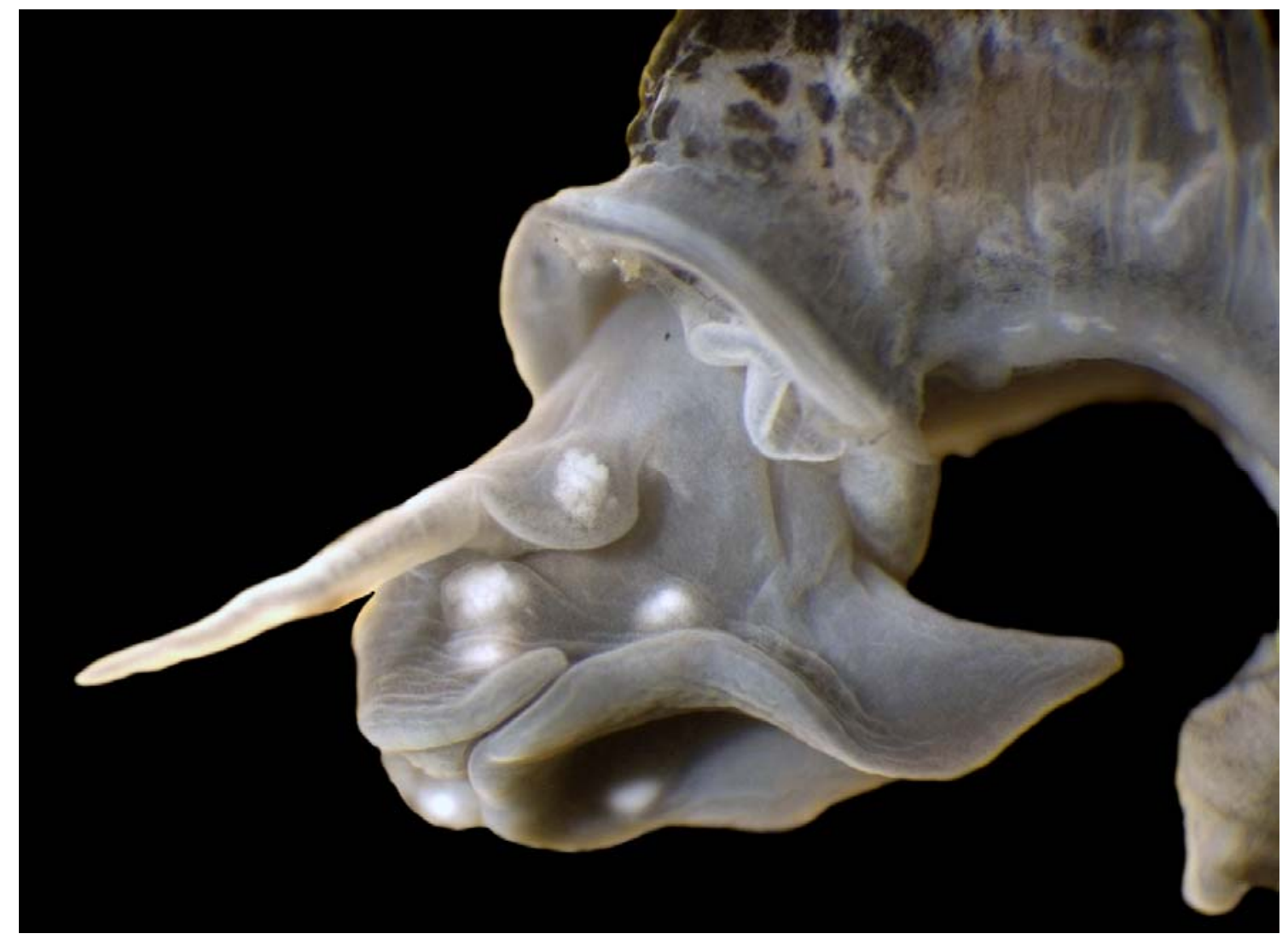

FIG. 1 

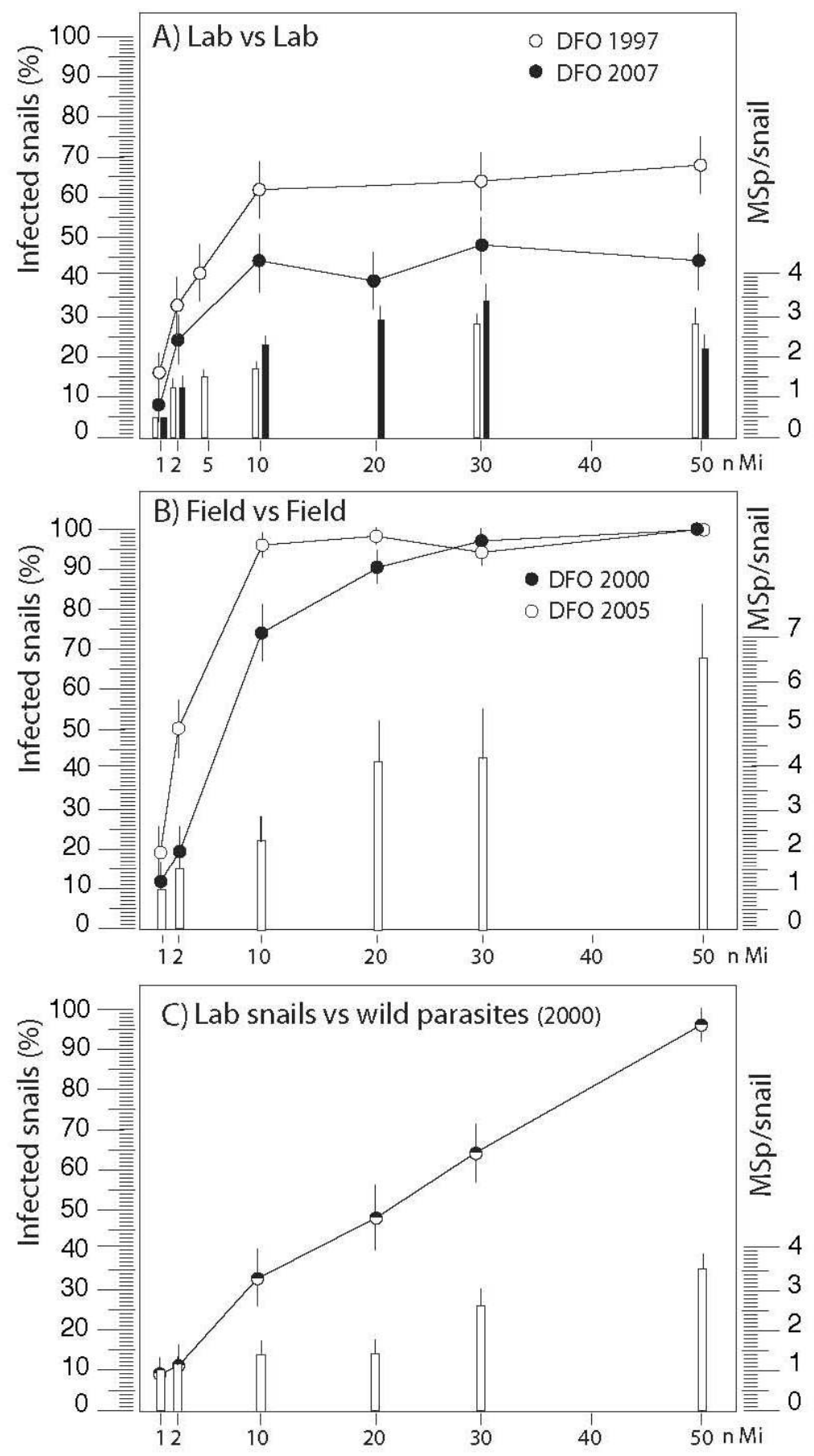

FIG. 2 
601

602

603

604

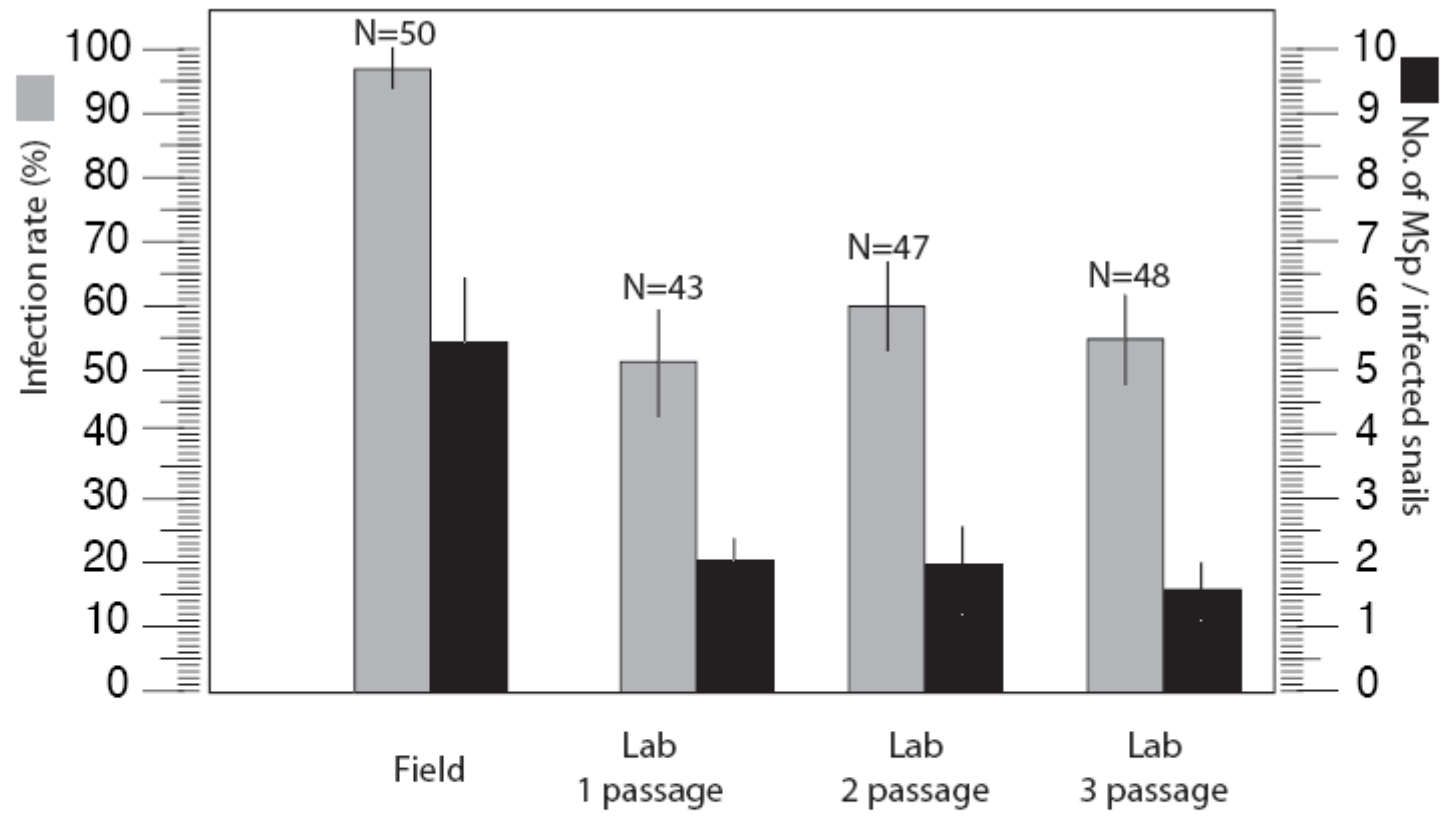

FIG.3

605

606

607 
608

609

610

611

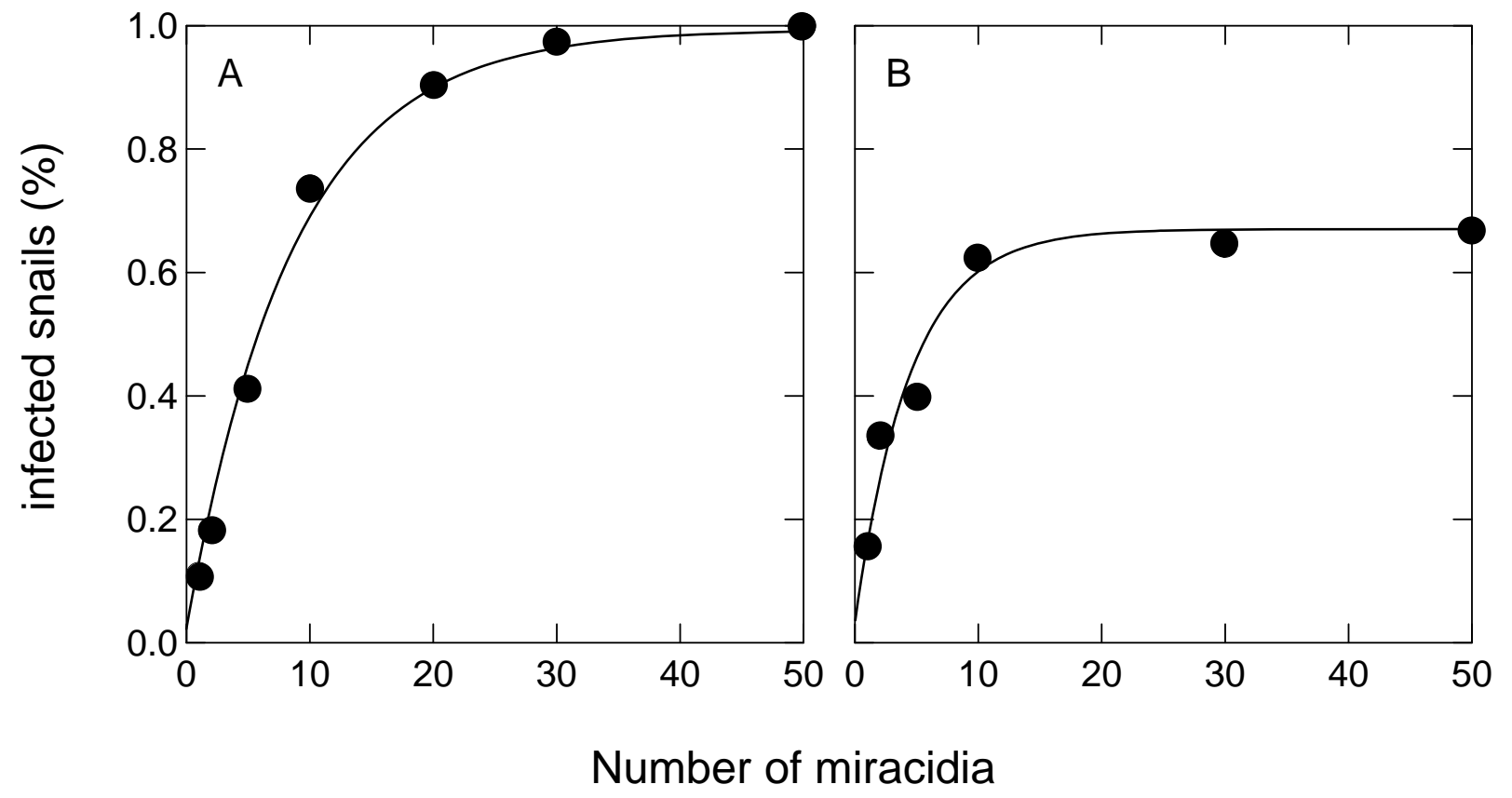

FIG. 4

613 


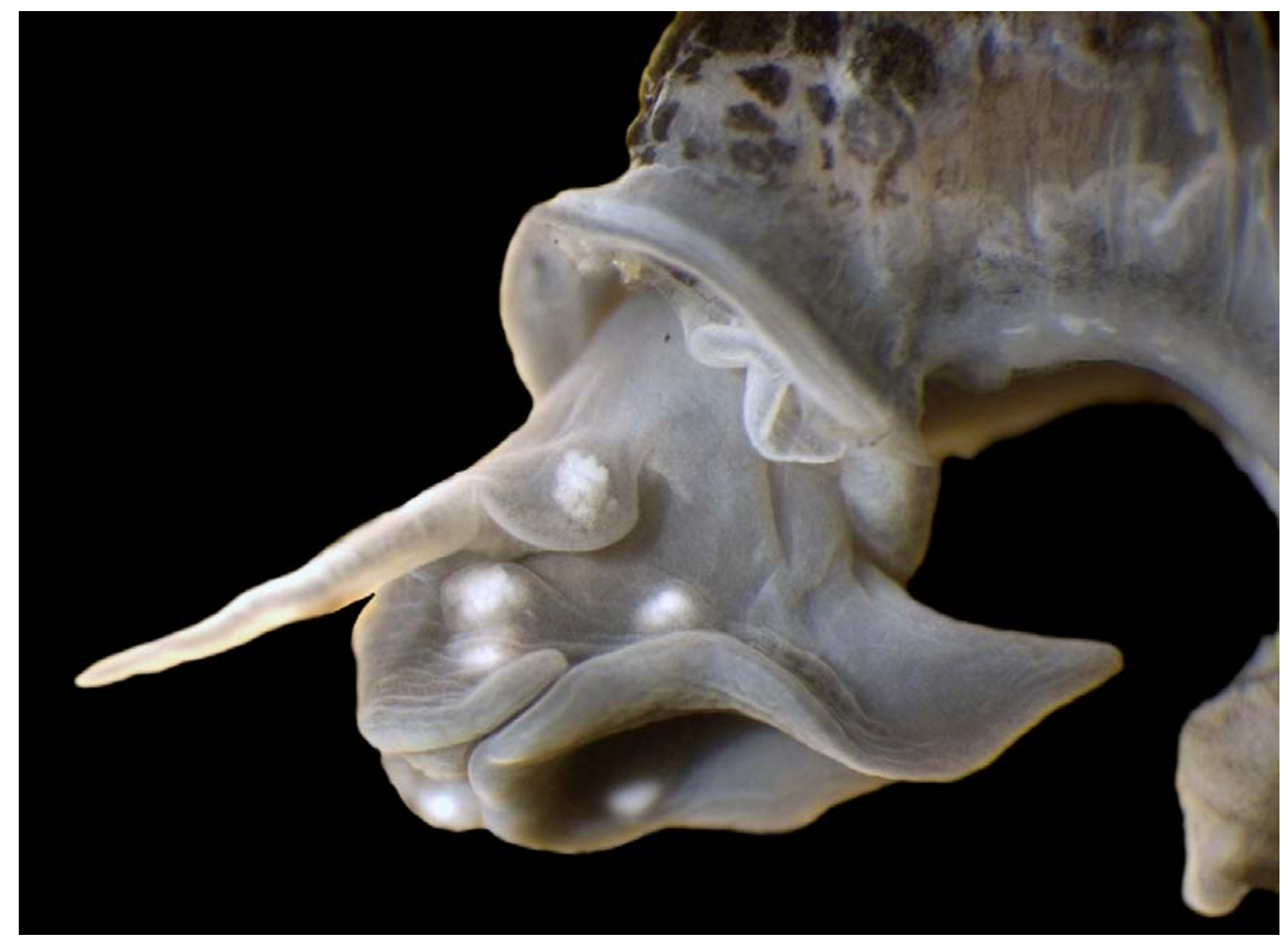

FIG. 1 

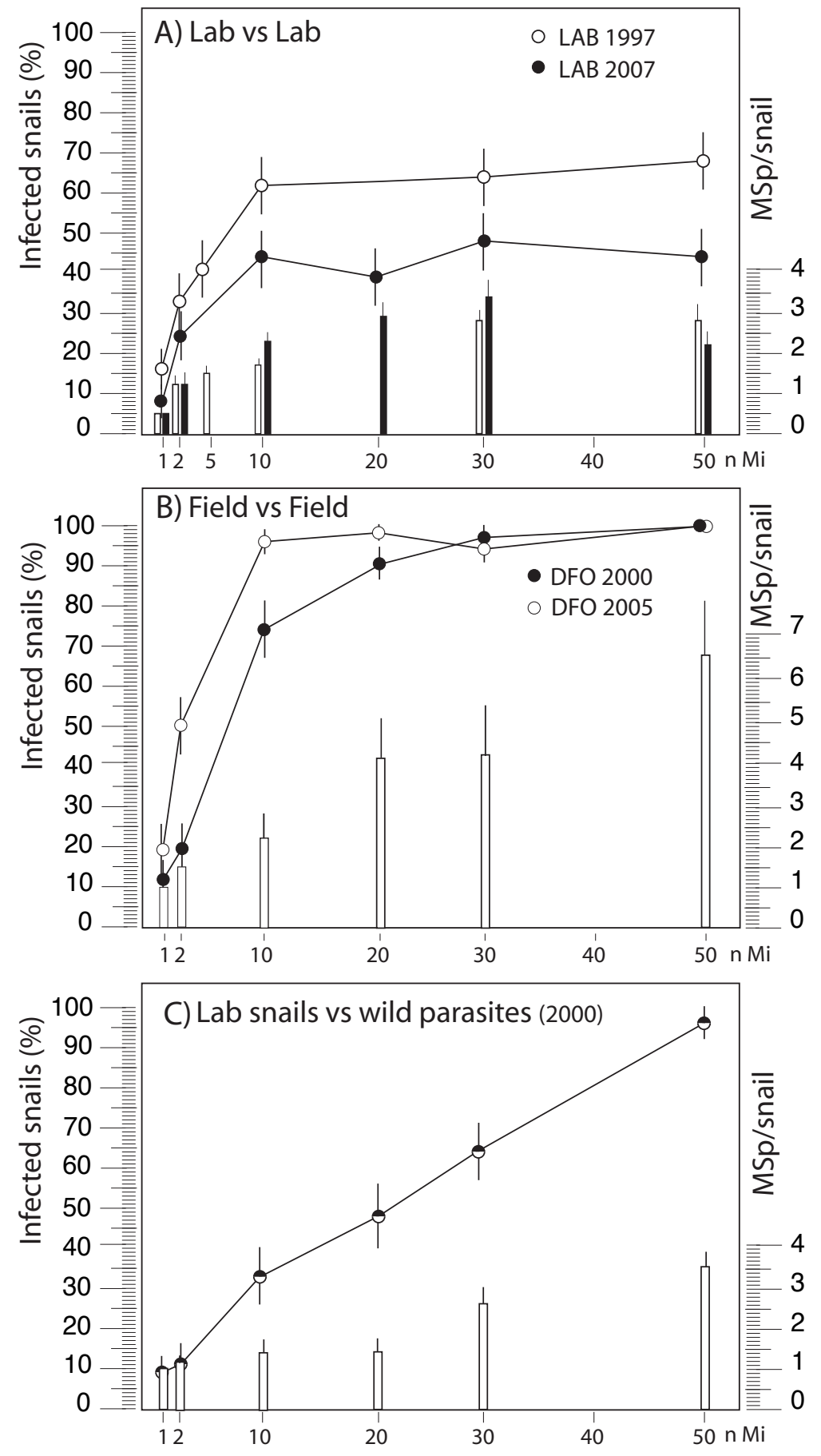

FIG. 2

MS ID: PAR-2008-0080.R1

Title: Effects of laboratory culture on compatibility between snails and schistosomes 


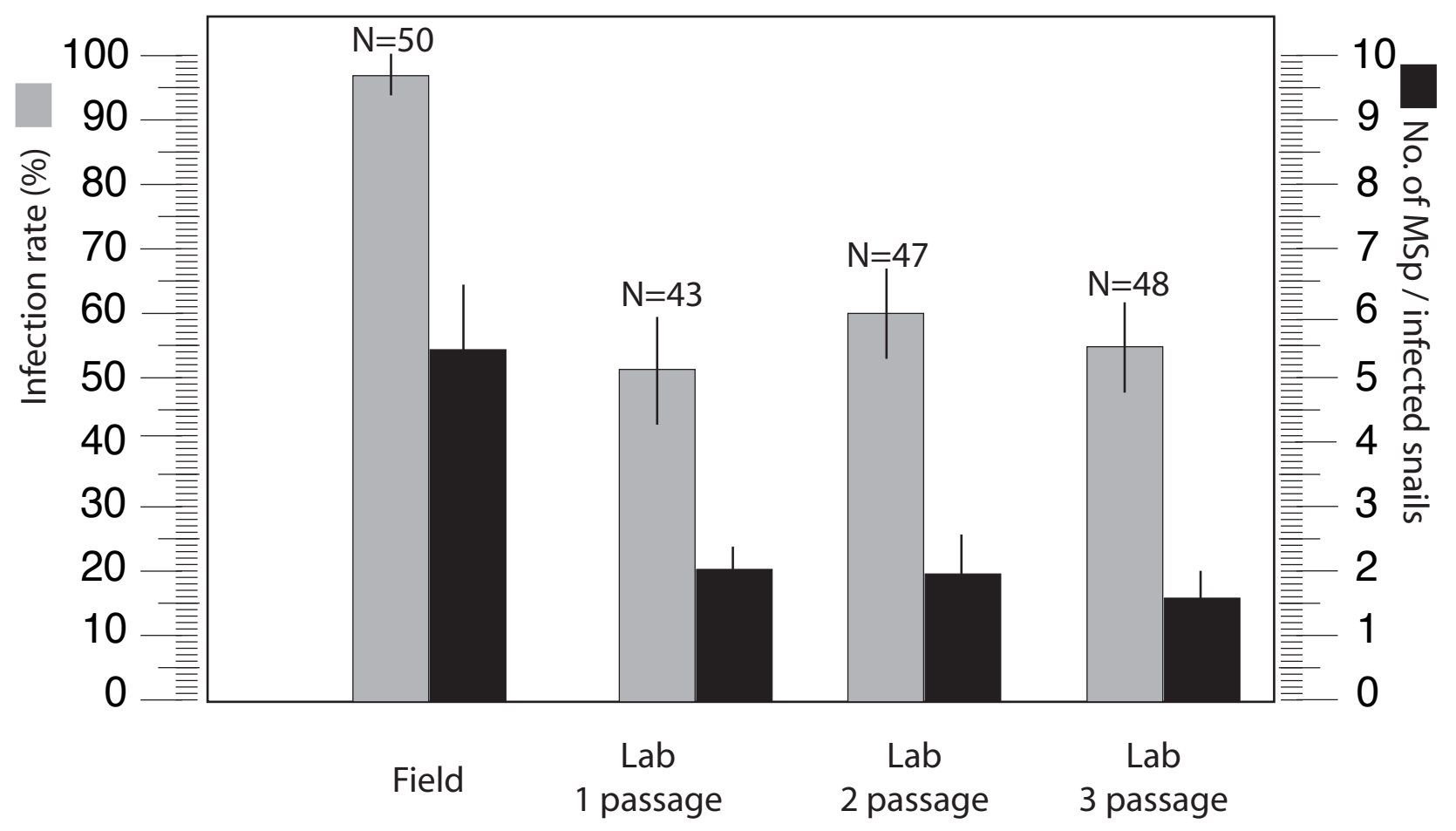

FIG.3

MS ID: PAR-2008-0080.R1

Title: Effects of laboratory culture on compatibility between snails and schistosomes 


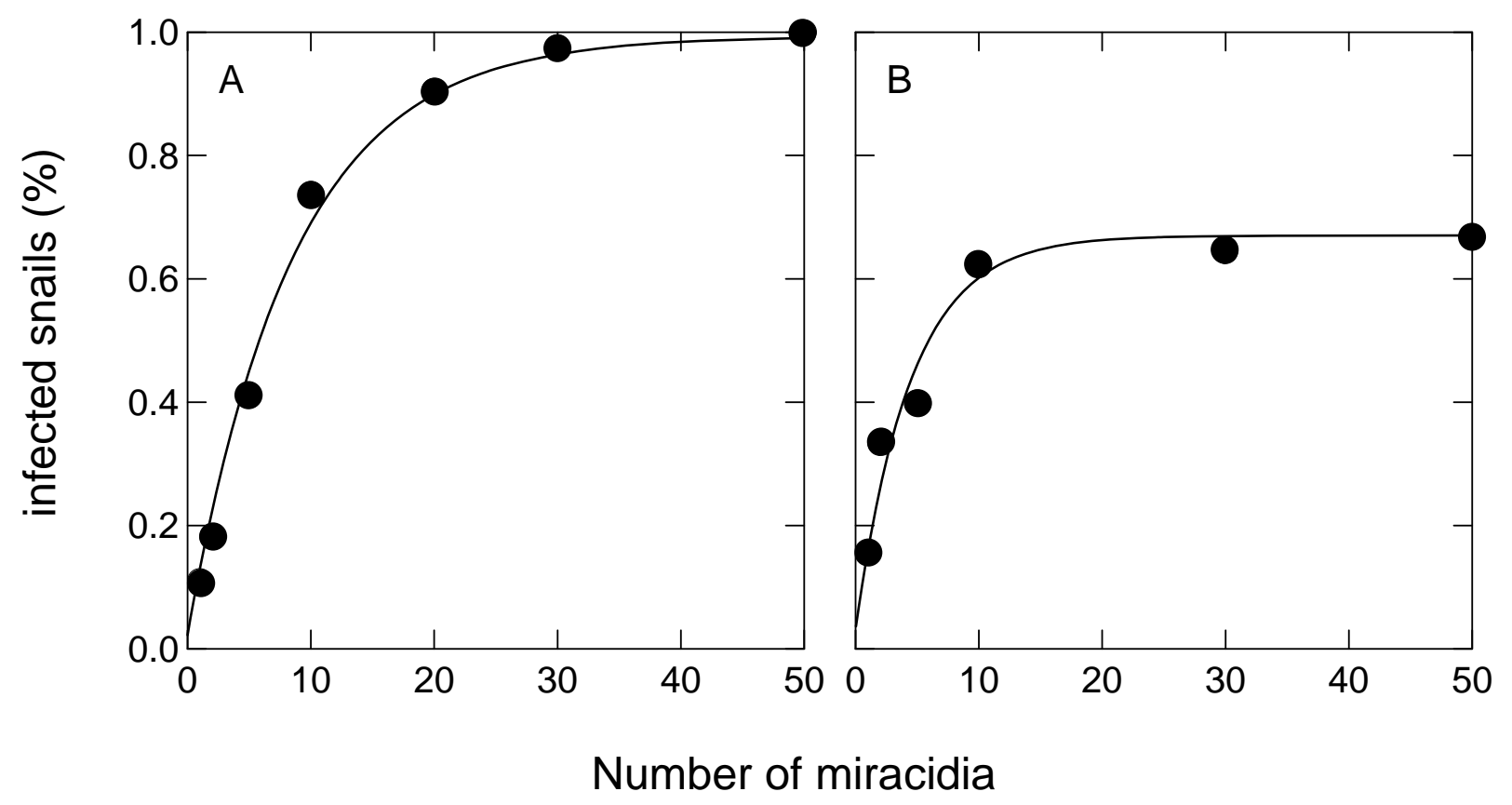

FIG. 4

MS ID: PAR-2008-0080.R1

Title: Effects of laboratory culture on compatibility between snails and schistosomes 